\title{
Implementation of Navigation System Available for Marine Leisure Ship*
}

\author{
Byoung-Guk Min ${ }^{1}$, Hee-ja Jeong ${ }^{2}$, Tae-Jin $\mathrm{Ha}^{2}$, Joon-Seub $\mathrm{Cha}^{3}$, Nam-Ho Kim ${ }^{3}$ \\ ${ }^{1}$ BonCInnovators Co., LTD., Korea \\ ${ }^{2}$ DongHaTech Co., LTD., Korea \\ ${ }^{3}$ Dept. Of Internet Contents, Honam University, Korea
}

\begin{abstract}
In this study, a marine navigation is proposed in order to solve problems including deviation from the route while a marine leisure ship is sailing, which gives a prior guide about expected route deviation at a real time through searching or makes it returned to the main routes from deviated areas when it is deviated from the navigated route. The proposed system is set with main routes and auxiliary ones. The latter provides the former with a prior guide about positions tended to be deviated while a ship is being sailed. Therefore, it has both of the functions to prevent route deviation in advance and to guide and make a ship returned to main routes from deviated ones by measuring the position of the ship until auxiliary routes and main ones are the same when the ship is deviated from the route.

Also, the proposed system can increase convenience and safety of a marine leisure ship by reducing the time for the route search server to search the routes from the deviated positions to the destinations and to transmit the information about them.
\end{abstract}

Keywords: power boat, path deviation, navigation, GPS, electronic marine chart, marine information

\section{Introduction}

USA and Europe- both private and personal power boat and yacht industries are constantly active. The above mentioned countries are making high value product by accumulated Technology in the small and medium size power boat industry. South Korea has sufficient IT technology but all those technology are not even used for the small and medium size power boat industry. South Korea's GDP has over \$22000; accordingly, power boat and yacht demand are increasing but high value products are being importe from US and Europe. In terms of automobile, IT technology for car is becoming a mandatory since car navigation has started its distribution and the user-variety-requirements-satisfied products are released. With this, leverage use for Digital Ship Navigator for small and medium sized power boats are grafted with a reliable "embedded hardware", "mapping technical" and "terrestrial ICT" technology programs that would be able to provide for an opportunity for growth and stagnancy of small and medium size power boat industry.

This marine navigation system development will result in the following impacts: for the safe navigation of the ship First, the ship must be installed of the navigation devices (AIS and RADAR, etc.) with the predicted collision Interface, and by implementing programs and danger alarms, it will provide early collision between ships that would enable to prevent accidents, Secondly, due to the dangerous goods in advance, it would be able to prevent

* This thesis is a research result that receives '2012 Honam Economic Region Strategic Industry rearing enterprise (Project Management No: 2012-0616) 
accidents in the sea by size power boat industry [1].

This marine navigation system development will result in the following impacts: for the safe navigation of the ship First, the ship must be installed of the navigation devices (AIS and RADAR, etc.) with the predicted collision InterBase, and by implementing programs and danger alarms, it will provide early collision between ships that would enable to prevent accidents. Secondly, due to the dangerous goods in advance, it would be able to prevent accidents in the sea by providing reminders and risk indicator for utilizing real-time of the ship's position and by orienting the extract information of the dangers from electronic chart based on the international standard 'S-57'. Third, shipping accidents and loss of life caused by pollution and protecting the public property will be expected by the general public welfare. Fourth, unlike conventional marine navigation equipment, it will maximize the user's convenience and smart devices by diffusion through the use of marine navigator and will enable the industry to target small and medium-sized ship with increased utilization.

(Figure 1 is shows the actual components of marine navigation. This provides real time information to the user of his own boat and other boat after interface of various support equipments for sailing upon installation and operation of boat. This large vessels are operating in the electronic chart display installed (ECDIS: Electronic Chart Display \& Information System) for real-time route planning and route monitoring include key features such as [2-4].)

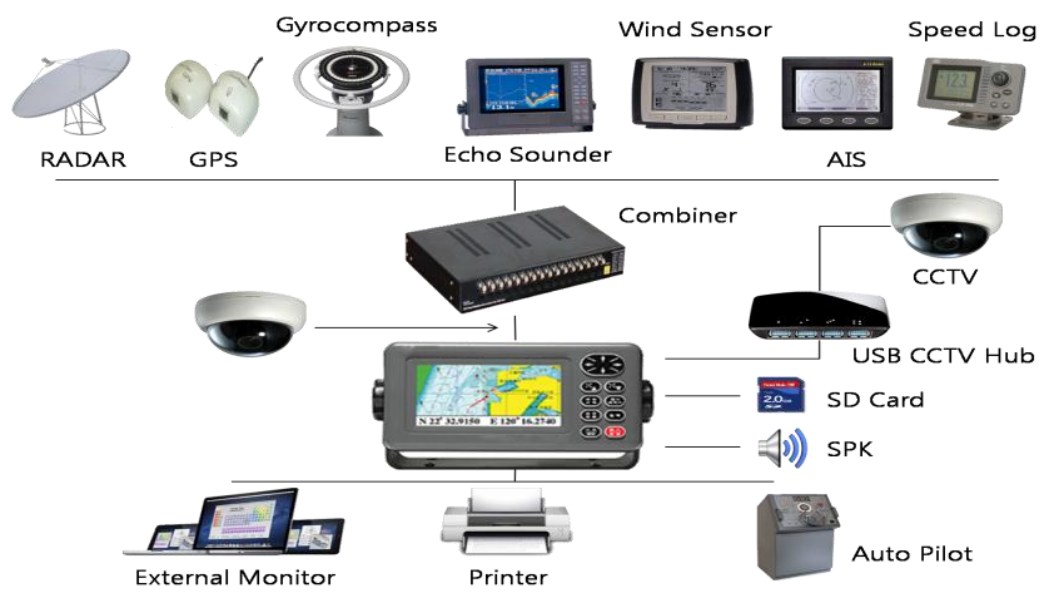

Figure 1. Marine Navigation Components

Recently, different from the past economic activity with the sea were increased quantitatively and a large ship operation of international trade was visible. However, small and medium size boat captains are worried about bumping each other more than big boats do in which route from fishing ship and yacht demand are increasingly complicated and diversified. As a result, the use of recreational craft is increased aside from the fact of the people's in familiarity of driving. Hence, the public demand for marine navigation is increased.

Original navigation devices are organized from devices of a car's navigation system that dedicated such device or mobile device including GPS used position data to check.

This treatise were designed about navigation for installed to small and medium-sized ships, marine navigation were designed to be used by routing and guidance to the point of arrival of land in marine applications. also, leaving a path on the sea voyage that may occur to solve the problem with a pre-departure course setting and guide navigation path and calculate expected 
to show a real sailing ship deviated from the main course main course at the point of departure when to return to the sub course set up to guide you through that increase ease of use and safety of marine navigation system is proposed.

\section{Technology Trend}

\section{1 Navigation}

Navigation of the main function is to provide search and guide most importantly, the essential device of the present position of the vehicle is sent from the satellite receiving micro wave is described by using a GPS receiver. GPS navigation receiver that is attached to receive signals from three or more satellites, and satellite navigation signals transmitted by the GPS receiver receives the incoming signal by measuring the time difference of the position and distance of each satellites and trilateration If you know how to use the three dimensional coordinate values obtained, produced by the coordinate values thus obtained on the Electronic Navigational Chart Matching present position if the value of navigation is displayed on the user's position [5-8]. (Figure 2 location based Electronic Navigational Chart of the course position is expressed.)

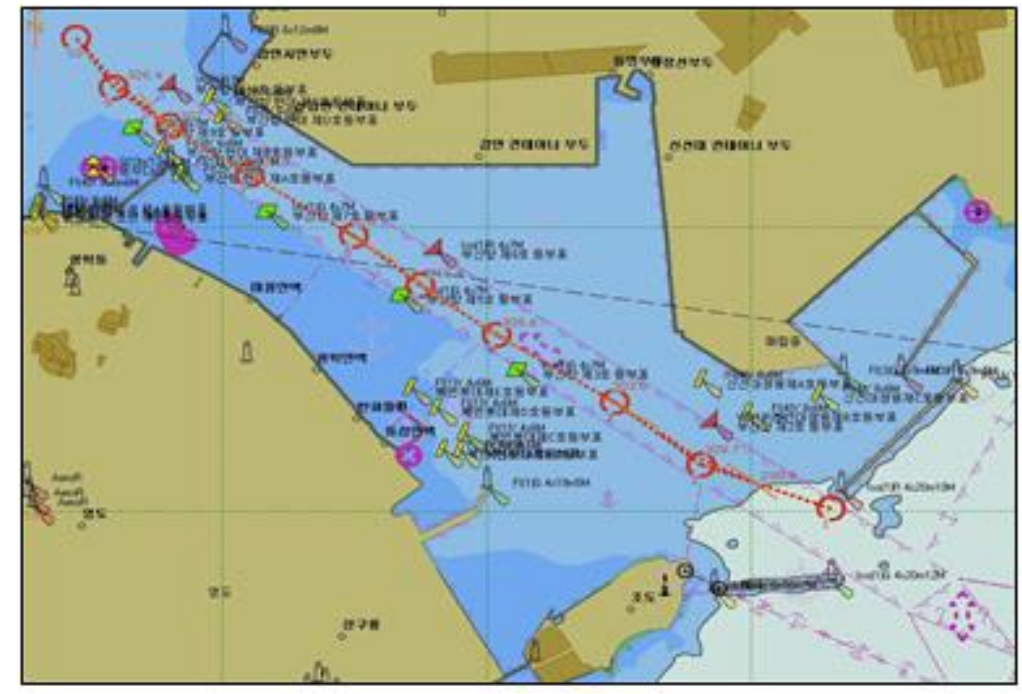

Figure 2. Course representation according to the location-based electronic marine chart

\section{2 Electronic Navigational Chart(ENC)}

IHO (International Hydrographic Office) Hydrographic Bureau in each country, as well as data exchange channel between the ECDIS manufacturers, navigator and electronic nautical chart to other users in order to supply GIS Data Exchange Standard as an international offshore marine GIS Standard Data of S-57 standard ENC is to be used.

ENC data file compatibility with other systems and data to leverage ISO/IEC8211 exchange binary data format consists of S-10x data because the data and navigation support data being produced of a pilot to convert separate data required in the form of text. It is through at first, ENC file to be read by designing ENC loader object and its attribute information of the file in text form is defined by converting 3D navigation support data format and the results are rearranged to fit its way of saving. (Figure 3 is Block Diagram for 
S-57 based ENC.)

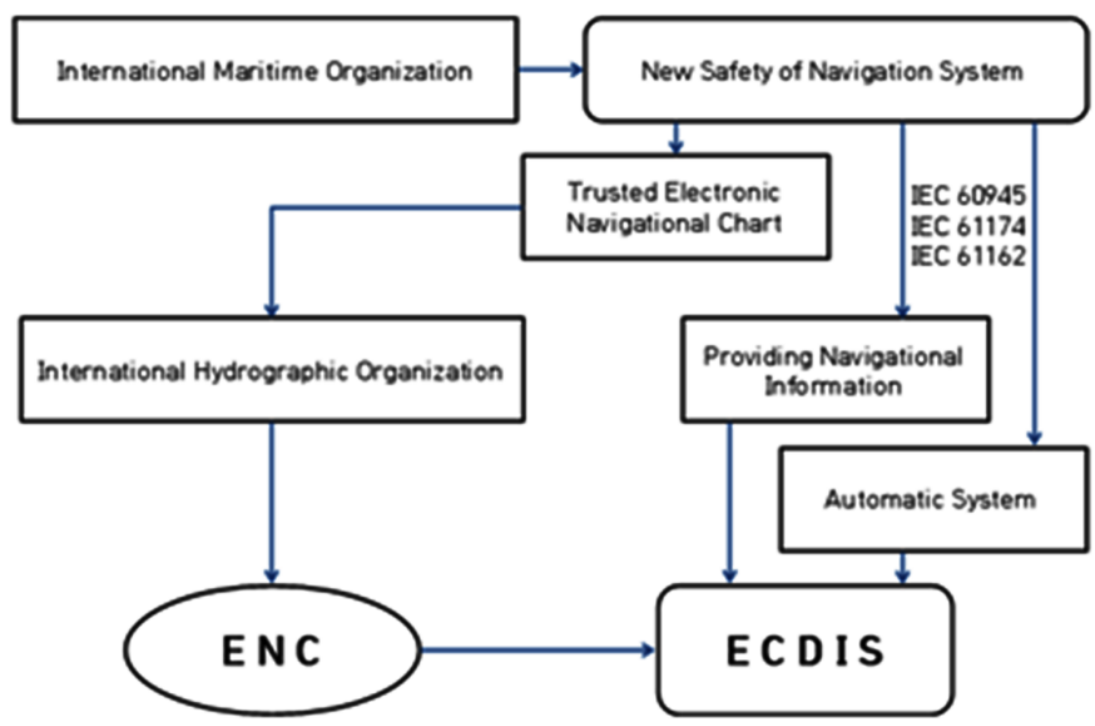

Figure 3. Block diagram of the S-57 based electronic marine charts

Figure 4 Screen representation flowchart of the electronic marine chart, and Figure 5 shows S57 and ISO/IEC8211 file format.

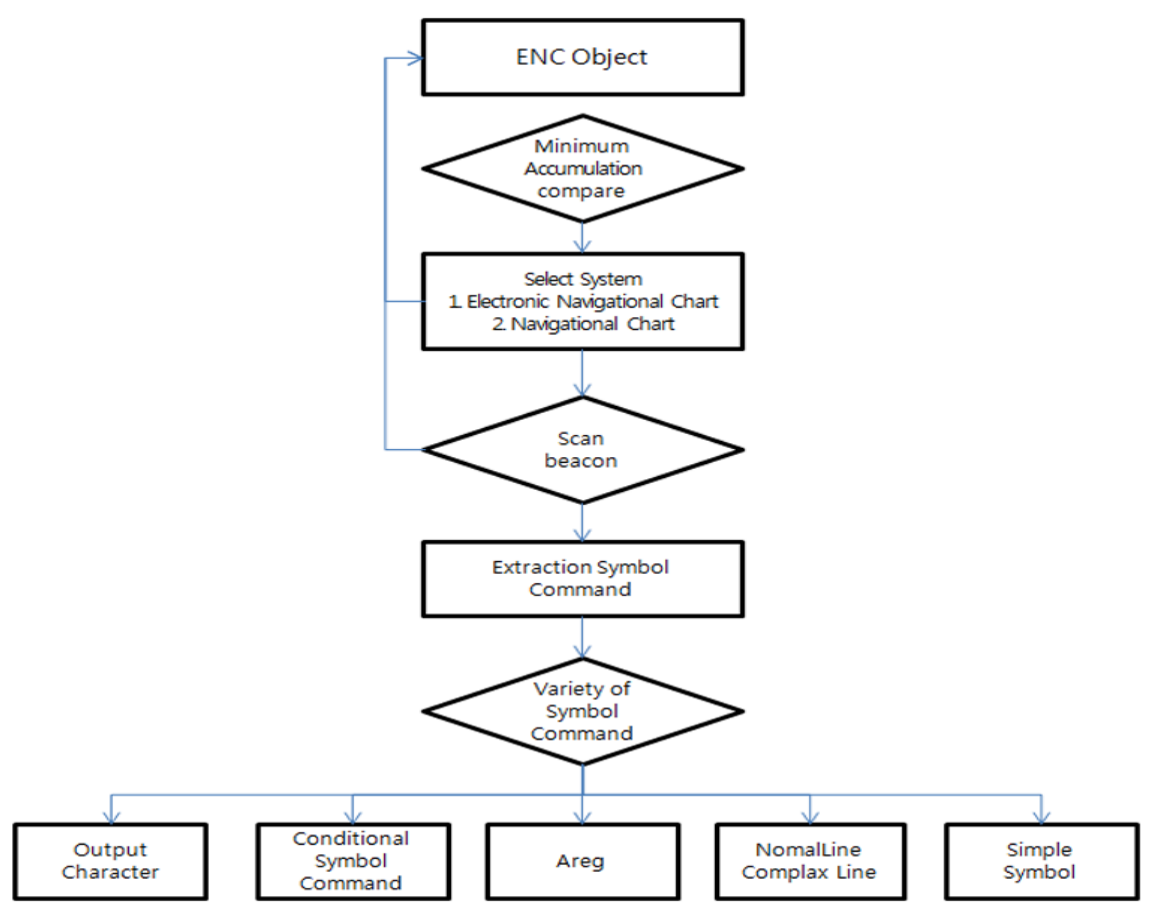

Figure 4. Screen representation flowchart of the electronic marine chart 

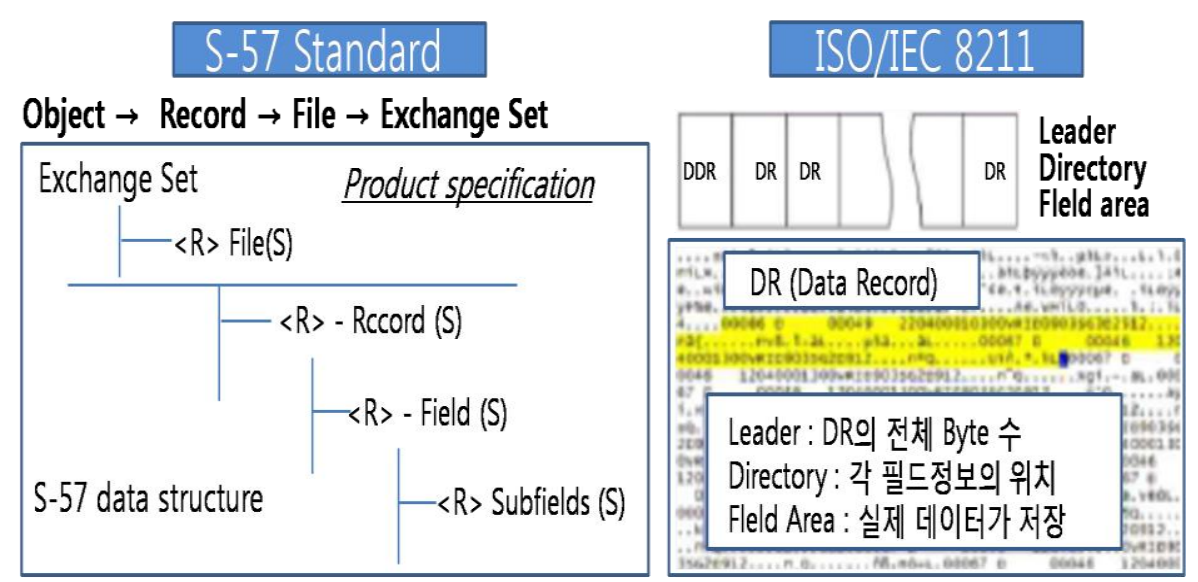

Figure 5. S-57 and ISO/IEC8211 file format

\section{Design of Marine Navigation System}

This thesis introduces new marine navigation that uses localization technology and duplex mobile network, and uses Telejmatics service. New marine navigation system's composition consist with controller, data transmitter, receiver, navigation sensor, voice processing, display, input, and memory.

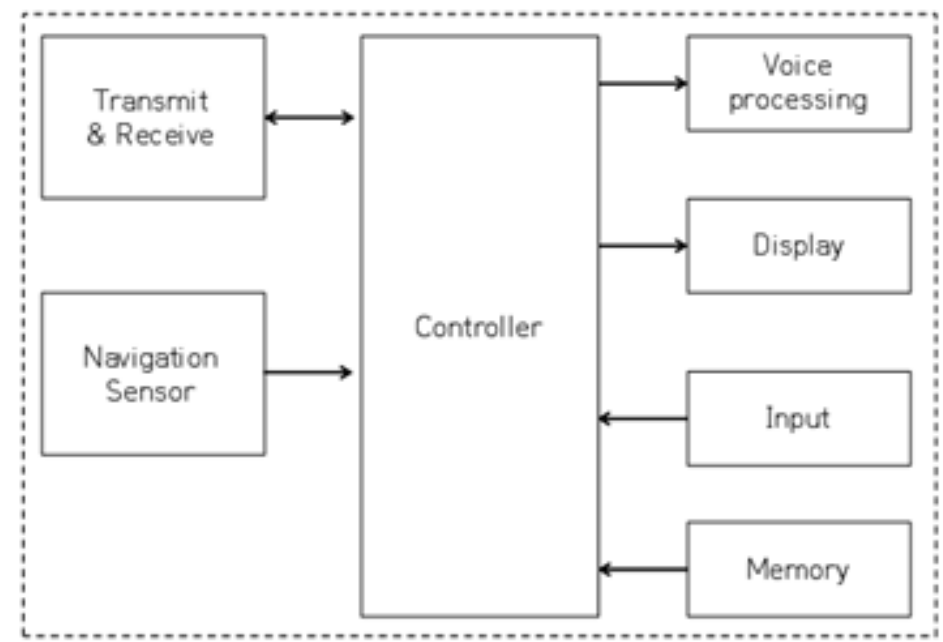

Figure 6. Block diagram for marine navigation

Marine navigation system of (Figure 6 Block diagram for marine navigation, Input, Memory, Server that searched for courses, Navigation Sensor, Transmit \& Receiver, and void processing and display.).

Object of navigation is an information of route. The purpose of these existing navigation and marine navigation that offer the same is, the function of the existing navigation system applied to marine navigation system shown in Figure 7 as implemented.

The existing marine navigation perform selecting departure and arrival, searching course, and calculating time after starting navigation system. It then starts to guide of course that uses voice and display. The ship is being operated by the GPS position in real-time check to make sure the right path to escape. Without creating path deviations during normal operation, it will 
continue until you arrive at your destination and perform route guidance. As soon as you arrive at your destination, the navigation system shuts down and exits. However, in the event of the departure of the course, it is on the system and re-route the request to search and present a new route to the destination from the point of departure and the search for a new navigation path calculation is performed to guide the course again.

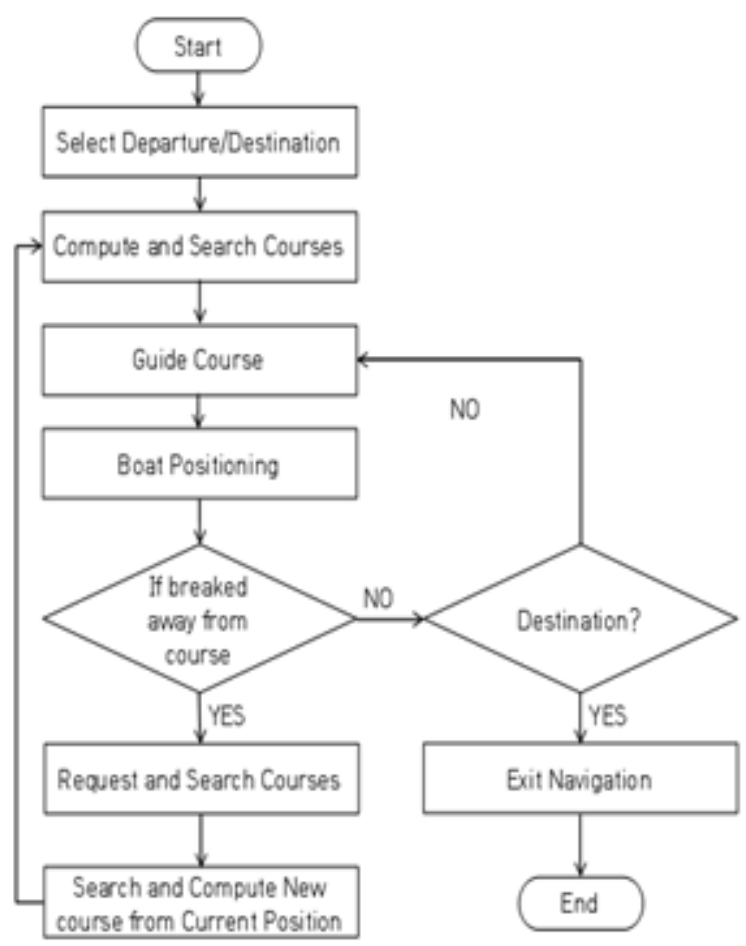

Figure 7. Marine navigation flowchart by the existing technology

As shown above, if you want to apply the existing navigation system on land at sea, sailing in sight to get a lot of information that is not much compared with the identification data is difficult.

Figure 8 Flowchart for a new marine navigation system. The proposed system is operating at sea, it's easy to lose the characteristic course to complement the system from the start and set the path to progress in the same way as the existing route guidance, but it before the main course guides the sub course. Operating in the sub course main course in advance of the departure point guide gives easy escape route in advance, with the ability to prevent secession, even if the path to return to the main course just provides convenience to a user plays an important role. While driving after that the main course will occur when the path deviate sub course, it will immediately return to the main course guide and shipping instructions and shipping to the operating position by measuring the sub course and the main course until a match to the main course to return sub course guidance is carried out. Airlines which sub course matches with the main course is then returned to the main course and check the position of flights become available after shipping arrives at your destination shall carry out the duties of navigation. 


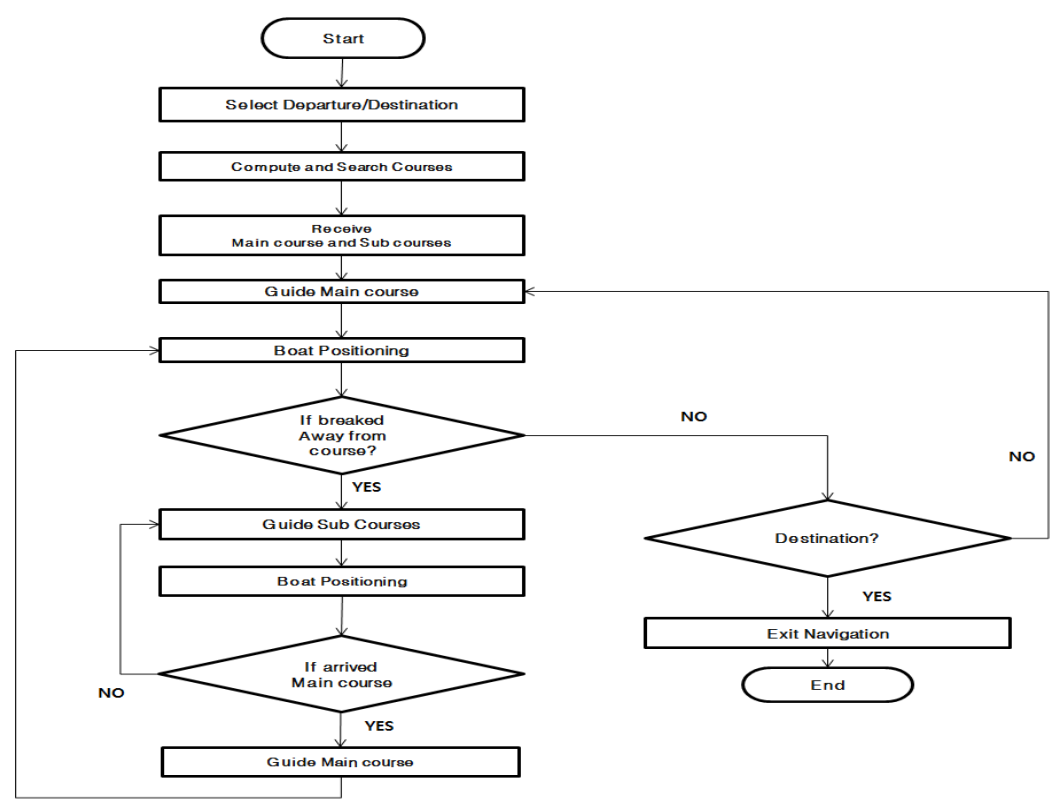

Figure 8. Flowchart for a new marine navigation system

\section{Conclusion}

This thesis suggests the marine navigation system. This system is warning abnormal course and shows optimum courses. Navigation system prevents breakaway, because of check wrong courses. And if the boat went wrong course, navigation system shows sub courses so that can go to main course. Because this system reduces required time that sends new search of course, this system contributes to advance user's usability and stability, and reduces financial burdens.

\section{References}

[1] J. W. Park, "A Proposal for Smart Future IT-Shipping convergence", Journal of The Institute of Electronics Engineers of Korea, vol. 35, no. 5, (2008) May, pp. 512-523.

[2] J. O. Baek, "A Study on the Implementation of Marine Network Standard for E-Navigation", Thesis of Master's degree Korea Maritime University, (2008) August.

[3] Y. H. Yu, "New trends of IT-Shipbuilding equipment Since NAV 54 Agenda", Weekly Technical Issue of National IT Industry Promotion Agency, The Ministry of Information and Communication, no.1368, (2008) October, pp. 29-38.

[4] K. Y. Kim, "A Study of Marine Network NMEA2000 for e-Navigation", Journal of the Korean Society of Marine Engineering vol. 34, no. 1, (2010) January, pp. 133-140.

[5] G. D. Lee, "[Navigation] Principle of Operation, Production process of Electronic map", Lifemaeil.com, Theme focus, (2008) December 11, http://www.lifemaeil.com/news_view.php?print_no=1321\&seq=21639.

[6] D. Budimir and F. Kamal, "Parallel coupled microstrip bandpass filter for X-band application", J. Science and Technology, Daffodil International Univ., vol. 2, no. 2, (2007) July, pp. 28-32.

[7] Z. A. Aghbari, "Studies on Modeling and Querying Video Databases", Ph. D. Dissertation, Kyushu University, (2001).

[8] Y. K. Chang and D. Y. Mum and S. J. Choi, "A Study of Ship Tracking System of GPS Using Marine Geographic Information Database", Korean Society of Civil Engineers, Korean Society of Civil Engineers Conference, vol. 2002, no. 11, (2002) November, pp. 2925-2928. 
International Journal of Control and Automation Vol.7, No.3 (2014) 